\title{
Mouse and supermouse
}

\section{from Jeffrey $G$. Williams}

THE mice displayed on the cover of this week's Nature are littermates but one has a bodyweight almost twice that of its sibling. They differ in that the larger mouse carries a hybrid gene containing the promoter of the mouse metallothionein-1 gene fused to the structural gene for rat growth hormone (see p.611). The 'transgenic' mouse was produced by injecting the hybrid gene into the pronucleus of a fertilized egg. Mice carrying the gene display very high levels of the fusion mRNA in their liver, high levels of growth hormone in their serum and an elevated growth rate. This is the first time that genetic engineering has been used to alter the phenotype of an animal in such a profound manner and is the culmination of a series of elegant experiments performed by Palmiter, Brinster and their colleagues over the past few years. There are almost certain to be great scientific and practical benefits from the application of genetic engineering techniques to animals. It is essential, however, to stress that there are limitations on what can be done - and more important, on what is worth doing.

When cloned genes are introduced into fertilized eggs by micro-injection a proportion of the mice that develop will carry the gene. The injected genes become chromosomally integrated but they are not found at the normal, nor even at a unique, location and the number of gene copies per cell also varies from animal to animal. The mice that develop from the injected eggs may also be chimaeras in which only some of the cells contain foreign DNA. However, a proportion of mice do transmit the newly acquired gene to their progeny and it $\mathrm{s}$ thus possible to produce mice containing the gene at a defined position in every cell.

The product of the mouse metallothionein-1 gene is a small cysteine rich polypeptide that binds heavy metals and is thought to be involved in zinc homeostasis and resistance to heavy metal toxicity. It is present in most tissues of the mouse, but the highest levels are found in liver. Transcription is inducible by heavy metals and glucocorticoids. Experiments reported earlier this year (Brinster et al. Nature 296, $39 ; 1982$ ) used a hybrid gene to establish that the sequence elements required for heavy metal inducibility lie within a stretch of DNA ninety nucleotides upstream of the start point of transcription. The hybrid gene contains a segment of the $5^{\prime}$ noncoding region and upstream sequences derived from the metallothionein-1 gene. Both were fused into the 5 ' non-coding region of the structural gene for herpes virus thymidine kinase to provide a readily assayable enzymatic activity. The hybrid gene was injected into fertilized mouse eggs which were incubated with cadmium as an inducer and then assayed for thymidine kinase activity.

The same fusion gene was used in two subsequent studies in which micro-injected fertilized eggs were re-implanted into foster mothers (Brinster et al. Cell 27, 223; 1981; Palmiter et al. Cell 29, 701; 1982). A high proportion of mice which carried the fusion gene expressed thymidine kinase in the liver. The activity was inducible with heavy metals but not with glucocorticoid hormones. For unknown reasons, in the progeny derived from these mice the expression of thymidine kinase was diminished or abolished in some cases and enhanced relative to the parent in others.

The metallothionein-growth hormone fusion gene was constructed using cxactly the same principle as the $t k$ fusion gene, with the genes being joined in their 5 ' noncoding regions. The mice contained variable numbers of copies of the gene and, with only one exception, the mice with the highest growth rate had the most copies of the gene. The mice were fed zinc to induce transcription of the fusion gene but this may not have been necessary. Most of the mice containing the gene were larger than normal before being fed zinc, and one animal which was removed from the zinc diet continued to grow at an accelerated rate. In mice with the highest copy number of the gene the level of growth hormone production was between 100 and 800 times the normal level. The serum concentration in one of the mice actually exceeded 100 rg $\mathrm{ml}^{-1}$.

Thus, aside from the variability of expression in second generation mice and the lack of response to glucocorticoids, results with the metallothionein promoter are highly encouraging. Sadly for the developmental biologist, however, this is not the case for globin genes. Although transcripts have been detected in muscle tissue from one mouse line carrying the rabbit $\beta$-globin gene, neither the rabbit $\beta$-globin gene, nor a fusion gene containing the $5^{\prime}$ half of the mouse $\beta$-globin gene and the $3^{\prime}$ half of a human $\beta$-globin gene are expressed in the blood cells of seven mouse lines carrying the rabbit gene and two lines carrying the fusion gene (Frank Constantini and Elizabeth Lacy, personal communication). This difference in behaviour of the $\beta$-globin genes and the metallothionein gene in transgenic mice may be a reflection of a fundamental difference between the two genes. Globin genes are expressed only in cells of the erythroid lineage and only at a specific stage in development. While expression of the metallothionein-1 gene varies from tissue to tissue, most tissues express the gene at some level. Thus metallothionein might almost be regarded as a housekeeping gene which displays tissue specific variation in the extent of expression. This in no way detracts from the usefulness of this promoter in obtaining high levels of expression of any gene to which it is suitably fused, and this is powerfully demonstrated by the growth hormone study.

The authors discuss a number of implications of their results. The elevation of serum protein levels by these means could provide a way of studying hormone action a way of accelerating animal growth, a method of producing valuable gene products and a means of correcting genetic disease. Before considering these last three applications individually there is one point which should be made. They all rely to a greater or lesser extent on the transmission through the germ line of the ability to express the gene. However, the metallothionein-1 thymidine kinase fusion gene was sometimes not expressed in the offspring of mice which werc themsclves expressing the gene. If the metallothioneingrowth hormone fusion gene behaves similarly this phenomenon will require further investigation.

The benefits which might accrue from a shorter production time seem a sufficient spur to ensure that fusion genes of this kind will be used to accelerate the growth of livestock animals. Also, the novel concept of genetic farming - the use of animals to produce large amounts of commercially important proteins - may be applied to the production of proteins which require secondary modifications not correctly made in yeast or other eukaryotic host cells. Finally, this achievement once again brings into question the potential of this technology as a means of correcting genetic disease. It will have become clear that such an approach will only be applicable where a genetic disorder may be prevented by simply elevating the serum concentration of a particular protein. In order to correct a genetic disorder such as a thalassemia by manipulation of the embryo, expression of the introduced gene would probably have to be limited to cells of the erythroid lineage and this is not at present achievable. Also, for reasons which have been presented in a recent review in this journal (Williamson, R. Nature 298, 416; 1982) it seems exceedingly unlikely that there will ever be a clinical requirement for such therapy to be applied to the embryo. Control sequences such as the metallothionein promoter may, however, prove useful in developing methods of correcting gene disorders in the somatic cells of affected patients.

Jeffrey G. Williams is at the Imperial Cancer Research Fund, Mill Hill Laboratories, Burtonhole Lane, London NW' IAD. 